\title{
PROYECTO DE REAL DECRETO DE CREACIÓN, RECONOCIMIENTO Y ACREDITACIÓN DE UNIVERSIDADES Y CENTROS UNIVERSITARIOS
}

\author{
Prof. Dra. Rosa Miren Pagola Petrirena \\ Aldezle de la Universidad de Deusto Prof. \\ Dr. José María Aguirre Oraá \\ Defensor de la Universidad de La Rioja
}

\section{Presentación y marco jurídico}

El Borrador del RD que aquí se presenta tiene como objeto "la regulación básica de los requisitos de creación y reconocimiento de Universidades y Centros universitarios públicos y privados, y el procedimiento para la autorización del inicio de sus actividades” (art. 1).

Viene a sustituir el RD 557/1991, de 12 de abril, "sobre creación y reconocimiento de Universidades y Centros universitarios" y a completar las leyes orgánicas, LO 6/2001, y su posterior modificación, LO 4/2007, en la primera de las cuales, en el art. 4.3., se recoge el objeto de aquel RD, acompañado de una referencia expresa a la calidad de la universidad en su totalidad: "Para garantizar la calidad de la docencia e investigación y, en general, del conjunto del sistema universitario, el Gobierno, previo informe del Consejo de Coordinación Universitaria, determinará, con carácter general, los requisitos básicos para la creación y reconocimiento de las Universidades. Los mencionados requisitos contemplarán los medios y recursos adecuados para el cumplimiento por las Universidades de las funciones a que se refiere el apartado 2 del artículo 1". El Borrador no cita el texto consolidado en la última modificación del 3 de febrero de 2015, obviamente, porque es posterior a la redacción del Borrador. Asimismo regula la acreditación institucional de centros universitarios, modificando el RD 1393/2007.

En resumen, siguiendo lo que dice en el preámbulo del Borrador, el presente RD tiene por objeto aclarar, simplificar y racionalizar los requisitos exigidos en la normativa vigente para la creación, reconocimiento y acreditación de las universidades. Pretende, además, contribuir a la creación de un entorno más favorable a la competencia y a la inversión, a la creación de empleo y de crecimiento en beneficio de los consumidores y usuarios, haciendo mención expresa de la Ley 20/2013 de garantía de la unidad de mercado. 
La transformación que se ha efectuado en las universidades españolas a partir de la implantación del Espacio Europeo de Educación Superior en España, exige la actualización de un RD que entró en vigor hace casi un cuarto de siglo y que es muy anterior a la LOU. El deseo de mejora en la calidad de la enseñanza, la investigación y, en general, del sistema universitario español, también hacía deseable una actualización a tenor del artículo 4.3 de la LO 6/2001 antes citado. No cabe, por tanto, más que felicitarse por la iniciativa. No obstante, puesto que el Proyecto está aún abierto a posibles aportaciones, convendría reflexionar sobre aquellos aspectos relevantes en orden a mejorar el servicio, aumentar la calidad y favorecer la gestión universitaria y su rendimiento para poder responder adecuadamente a las necesidades y exigencias de los nuevos tiempos que debe afrontar la universidad. Con esta intención, se presentan los comentarios que siguen.

II. Contenido y comentarios al Borrador de R. D. Análisis del articulado

\section{Objeto (Art. 1)}

El objeto de este RD, explicitado en el art. 1, "regulación básica de los requisitos de creación y reconocimiento de las Universidades y Centros públicos y privados, y el procedimiento para la autorización del inicio de sus actividades”, no se alcanza en su desarrollo, tal como se irá recogiendo en el análisis de los sucesivos artículos. Se solicita, en consecuencia, una regulación íntegra de todos los aspectos que aborda.

Denominaciones (Art. 2)

El punto 3. indica que "No podrán utilizarse denominaciones que por su significado puedan inducir a confusión con las Universidades...”.

Para el adecuado cumplimiento de este artículo, el RD debería establecer un instrumento jurídico para su aplicación efectiva y reglamentar un procedimiento corrector a quienes utilicen denominaciones contrarias a lo previsto en el artículo.

Requisitos de las universidades (Art. 4)

Los recursos y requisitos previos para prestar el servicio público y desarrollar sus funciones quedan recogidos del siguiente modo:

a) Disponer de una oferta académica mínima de titulaciones oficiales.

b) Contar con una programación investigadora adecuada.

c) Disponer de personal docente e investigador en número suficiente y cualificado. 
d) Disponer de instalaciones y recursos adecuados para el cumplimiento de sus funciones.

e) Contar con una organización y estructura adecuada.

f) Garantizar la prestación del servicio.

g) Garantizar que sus Estatutos, régimen jurídico y normas de organización y funcionamiento, conformes a lo establecido en la LO 6/2001.

Y su desarrollo queda recogido del siguiente modo: a) y b) "Actividad docente e investigadora” (art. 5); c) "Personal docente e investigador" (art. 6); d) "Instalaciones" (art. 7); e) “Organización y funcionamiento” (art. 9); f) Garantía de la actividad (art. 8).

Actividad docente e investigadora (Art. 5)

Respecto a la oferta (5.1), se mantiene el número de títulos de carácter oficial exigidos: 8 títulos de grado y/o máster. No hay ninguna obligatoriedad para el modelo de oferta, a diferencia del RD 557/1991 que obliga a que no menos de tres impartan el segundo ciclo y, al menos, una de éstos, de Ciencias Experimentales o estudios técnicos” (art. 5,1).

Igualmente, a diferencia del RD 557/1991, tampoco se especifica nada acerca de que: "se tendrán en cuenta las necesidades de programación general de la enseñanza en su nivel superior, derivadas de la población escolar, del desarrollo de nuevas ramas surgidas del avance científico y las necesidades de los distintos sectores profesionales, así como su incidencia en el entorno geográfico, de acuerdo con la normativa vigente en materia de planificación urbanística” (art. $4^{\circ}$ ).

Estos dos aspectos han sido interpretados como concesión a las Universidades privadas, tanto por los sindicatos como por algunos medios de comunicación.

En lo que concierne a la investigación no hay nada que comentar, puesto que sólo indica que se debe promover el desarrollo de dicha actividad y la obligatoriedad de presentar, para la acreditación, una programación plurianual de la actividad investigadora. También, al igual que en el RD 557/1991, se exige la impartición de las enseñanzas de doctorado.

La acreditación de los requisitos previos debe ser tramitada mediante la presentación, además de la programación plurianual de la actividad investigadora, citada arriba, de:

1. Un plan de desarrollo de titulaciones por cada rama de conocimiento haciendo constar:

La relación de titulaciones

La previsión del número total de plazas 
El curso académico en que darán comienzo las actividades

Calendario de implantación enseñanzas, funcionamiento de los centros

Medios dispuestos para su desarrollo

El plan debe ser evaluado por la ANECA o la agencia que la Comunidad Autónoma determine. La implantación se supedita al procedimiento de verificación y acreditación de los planes previstos en el RD 1393/2007.

El punto 2.a), que trata sobre la evaluación de las agencias de evaluación del plan de desarrollo de las titulaciones, no fija los criterios mediante los cuales deba procederse a la evaluación, lo que supone un control difuso que genera una gran inseguridad jurídica. Por otra parte, excede en las funciones atribuibles a un órgano de esta naturaleza y, en consecuencia, invade las competencias que corresponden a las Administraciones educativas que residen en las CCAA.

Personal docente e investigador (Art. 6)

1. La ratio establecida entre profesor / alumno es de $1 / 25$, entendido como personal docente e investigador computado en régimen de dedicación a tiempo completo o su equivalente a tiempo parcial. Esta cifra deberá modularse en la enseñanza no presencial.

2. En cuanto a la composición:

Título de grado: $50 \%$ doctores

Título de Máster: 70\% doctores

Enseñanzas de doctorado: 100\% doctores

3. Dedicación a tiempo completo en Univ. Públ. o régimen similar, al menos el $60 \%$ en las Univ. Priv.

4. Compatibilidad del profesorado, tanto en las Univ. Públ. como en las Univ. Priv., según LO 6/2001, art. 72.

5. Para la acreditación de los requisitos, se debe aportar la plantilla del personal docente e investigador al comienzo de la actividad y la previsión de su incremento anual hasta la implantación de las enseñanzas.

- Respecto a la normativa vigente, RD 557/1991, se mantienen las ratios de profesor/ alumno. Ha aumentado la exigencia en el número de doctores para los grados $50 \%$ Borrador, 30\% enseñanzas primer ciclo RD 557/1991, art. 7, a). La obligatoriedad de que al menos el 50\% del profesorado debe ser doctor ya estaba establecida en la LO 4/2007, 
setenta y cuatro. El 70\% de doctores en los segundos ciclos, RD 557/1991Art, b), coincide con la exigencia establecida para los estudios de Máster del Borrador y del título de doctor en la totalidad del profesorado del doctorado (art. 7, c).

- Los porcentajes señalados en los apartados 2 y 3 debieran explicitarse referidos en "equivalente a tiempo completo" y tomando como referencia el "mínimo legal exigible", es decir, la ratio $1 / 25$, tal como aparece en el apartado 1 . Quiere decir que el porcentaje debe venir referido al profesorado que resulte exigible legalmente (llamada ratio teórica 1/25), de modo similar a como se contempla en la disposición transitoria única. Tal como está puede dar lugar a que la universidad mejore la ratio de profesorado del punto $1^{\circ}$, pero que incumpla más fácilmente las ratios de los apartados 2 y 3 .

- Sería conveniente definir cómo se computan los profesores a tiempo parcial para hacer los cálculos de profesorado y, de este modo, determinar las ratios que se piden.

- En cuanto al número total de profesores con el título de doctor, se mantiene, la modificación setenta y cuatro hecha al art. 72, 2 de la LO 6/2001 en la LO 4/2007, que lo fija en un mínimo del $50 \%$ y, al menos, el $60 \%$, de la totalidad de su profesorado doctor debe contar con la evaluación positiva de la ANECA u otra agencia de evaluación externa.

- Los porcentajes que se señalan para el número de doctores por tipo de enseñanza resultan discutibles. Especialmente, la exigencia del $70 \%$ de doctores para los másteres y más todavía en aquellos cuya orientación es la especialización profesional. Convendría, en todo caso, distinguir los que tienen orientación preferentemente académica o los de iniciación a la investigación, distinción que sí se recoge en el RD 1393/2007, art. 10 y también en las guías para la verificación de títulos de la ANECA. Este requisito puede suponer que se siga estableciendo la equiparación entre los segundos ciclos de las licenciaturas con los másteres, pero no es adecuada, y más teniendo en cuenta el gran número de másteres profesionalizantes que se imparten. En consecuencia, debieran revisarse los porcentajes y aplicar el cómputo según el tipo de enseñanza.

- En cualquier caso, debiera de garantizarse que esta exigencia no será tomada como criterio de evaluación por parte de las agencias de evaluación y que su comprobación le corresponda únicamente a la administración educativa competente.

- Respecto a la limitación de que el 60\% del profesorado ejerza sus funciones a tiempo completo, punto 3, por parte de las universidades privadas, además de solicitar que debería calcularse sobre el resultado de aplicar la ratio mínima de $1 / 25$, habría que tener en consideración los siguientes motivos: 
- No se halla explícitamente recogido en un texto normativo con rango de ley.

- No es realista y es contrario a la autonomía universitaria imponer las condiciones laborales de dedicación y contratación del personal docente.

- Se limita a las universidades contratar profesionales en activo de sectores vinculados a las enseñanzas universitarias.

- Se impone a las universidades privadas una estructura de costes salariales similar a la de la universidad pública, vulnerando las normas del derecho de la competencia y la ley de unidad de mercado.

\section{Instalaciones (Art. 7)}

Las infraestructuras y medios materiales mínimos exigidos en el Borrador son:

-Espacios docentes e investigadores. Los módulos orientativos vienen especificados en el Anexo 1. Los espacios que se regulan comprenden: Aulas. Laboratorios docentes -separados de aulas y salas de tutorías-. Laboratorios de investigación. Despachos de profesores. Seminarios. Cada uno está regulado por el número de metros que debe disponer en relación con el número de alumnos, el mobiliario, la disponibilidad y flexibilidad exigida, entre otros requisitos.

-Centro de Recursos para Aprendizaje y la Investigación (CRAI). Comprende los servicios, tanto físicos como virtuales, entre los que se incluye la biblioteca. En su conjunto debe permitir la utilización simultánea de, al menos, el 10\% de alumnos matriculados.

-Equipo informático: aulas, servicios generales, con conectividad a la red. No regula el número de ordenadores por estudiante, sólo indica "el número adecuado". El acceso a los requisitos docentes y científicos, vía servicios web, también debe garantizarse.

- Las exigencias adicionales destinadas al ámbito de las Ciencias de la Salud se establecen en el anexo 2. y 4. Aluden de modo general a la exclusividad del uso académico; el cumplimiento de la legislación vigente en cuanto a las condiciones de prevención de riesgos laborales, acústicos y de habitabilidad, así como a las condiciones arquitectónicas que requieren el acceso y movilidad de las personas con discapacidad.

- El punto 1 de esta regulación, de aplicación tanto para las universidades públicas como para las privadas, presenta algunas modificaciones. Se ha reducido la exigencia establecida para los Laboratorios docentes, de los 7 metros cuadrados por alumno exigidos se ha pasado a 5. También se ha flexibilizado la exigencia establecida para los Laboratorios 
de investigación, de los 15 metros cuadrados por profesor o investigador actuales a la franja entre 10 y 15. Los seminarios han ampliado la exigencia, de los 2,5 metros cuadrados por alumno matriculado se ha pasado a 3, "garantizando un mínimo de uso simultáneo por parte del 5\% de los alumnos”.

- Actualiza y adapta a las necesidades tecnológicas, de modo muy general, el apartado destinado al CRAI, únicamente Biblioteca en la normativa actual, definiendo el espacio mínimo necesario: utilización simultánea de, al menos, un 10\% del número total de alumnos matriculados.

- No hay diferencias esenciales en lo referente a las exigencias para las enseñanzas en el ámbito de las Ciencias de la Salud, tanto en lo concerniente a las universidades públicas como a las privadas. Corresponde a los Ministerios correspondientes, Educación y Salud, el establecimiento de los requisitos referidos a la dotación e instalaciones de las Instituciones Sanitarias, como en la LO 6/2001, Disposición adicional séptima. Puntualiza la denominación de "Hospital Universitario" y establece la vía de los convenios con instituciones, tanto públicas como privadas, para garantizar la disponibilidad de los medios clínicos necesarios para las enseñanzas de Odontología, Podología, Psicología, etc.

- Siguiendo el espíritu renovador del EEES, que fomenta la docencia, en especial de ciertas titulaciones, en las entidades colaboradoras, hospitales, centros tecnológicos, centros de innovación, startups, etc. se propone un cambio en la redacción y sustituir: "las universidades y sus centros deben disponer de instalaciones destinadas exclusivamente a uso académico" por "las universidades y sus centros deben disponer de instalaciones adecuadas y compatibles con el uso académico”.

- Los requisitos de espacios, detallados en el Anexo 1, referidos a aulas, laboratorios, despachos, seminarios pueden ser muy exigentes, salvo que se calculen de nuevo en términos globales, de media y no elemento a elemento.

Garantía de actividad (Art. 8)

Para garantizar la continuidad de los centros, las universidades en vías de creación, tanto públicas como privadas, deben aportar:

a) Un estudio de viabilidad económica del proyecto. Las universidades privadas, además, entre los gastos previstos deberán incluir una partida con el porcentaje destinado a becas y ayudas (estudio e investigación). 
b) Las Universidades privadas también deben aportar garantías financieras que aseguren su financiación económica, proporcionales al número de títulos ofertados y de alumnos matriculados.

c) El compromiso de mantener en funcionamiento la universidad y cada uno de los centros durante el tiempo suficiente a fin de que permita finalizar los estudios a los alumnos.

- Aunque parece lógico que se pidan garantías financieras para asegurar el funcionamiento de la actividad universitaria, puede contradecir el art. 10 de la Ley de libre de acceso de Servicios y el art. 18 de la Ley 20/2013, de 9 de diciembre, de garantía de la unidad de mercado.

Inicio de actividades (Art. 10)

Lo esencial de este artículo de tres puntos, salvo la referencia a la documentación aportada, explicitada en el Anexo 3, queda reflejado en:

a) El órgano competente de la Comunidad Autónoma es el que autoriza el inicio.

b) El procedimiento de autorización se iniciará a solicitud del interesado y tendrá una duración máxima de seis meses.

- En el punto 1, hay una variación respecto al RD 557/1991, art. 15, en donde la formulación difiere entre las Univ. públicas y las privadas. La autorización de las públicas la realiza la "Administración educativa competente, previa homologación por el Consejo de Universidades de los correspondientes planes de estudios de las enseñanzas que se vayan a impartir" (art. 15, Uno). Mientras que la apertura de las privadas corre a cargo de la "Administración competente en un plazo no superior a seis meses, previa comprobación de que se han cumplido los requisitos adquiridos por la entidad y han sido homologados por el Gobierno los títulos oficiales a expedir por la misma” (art. 15, Dos).

- El Borrador atribuye a la Comunidad Autónoma la competencia de la autorización del inicio. Se unifica el criterio para los dos tipos de universidades; se suprime la comprobación de los títulos oficiales en las privadas, que ya queda regulado en el lugar correspondiente.

- El inicio de actividades de la universidad, una vez creada o reconocida, puede entenderse que se trata de la exigencia de una doble licencia para el ejercicio de la actividad universitaria: la primera, para crear o reconocer la universidad, y la segunda, para ejercer la actividad. 
- Acorde con los criterios establecidos en la Ley 20/2013, de 9 de diciembre, de garantía de la unidad de mercado, debería establecerse el procedimiento de "declaración responsable”, y entenderse concedido por silencio positivo.

Supervisión y control (Art. 11)

Las universidades deben presentar al órgano competente de la Comunidad Autónoma una memoria de sus actividades, docentes e investigadoras.

El órgano competente de la Comunidad Autónoma será el que, en caso de incumplimiento de los requisitos jurídicos de una universidad, requerirá su regulación. La evolución del número de estudiantes será criterio particular a tener en cuenta.

En caso de incumplimiento y, previa audiencia, la Administración podrá revocar la autorización de inicio de la actividad de la universidad en su conjunto o a alguno de sus centros.

- El art. 5 Tres del RD 557/1991 se recoge sin variación en el punto 1 del Borrador. En la legislación vigente, los puntos 2 y 3 del Borrador sólo se aplican, con algunas alteraciones, a las universidades privadas, LO 6/2001, Disposición adicional novena, punto 3 y RD 557/1991, art. 12.

- Este artículo contiene una disposición de mucha mayor importancia de la que podría parecer por su ubicación en la norma. De hecho, contempla el control periódico, de carácter anual, del cumplimiento de los requisitos exigidos e, incluso, puede llevar a la revocación de la autorización de la actividad, por tanto, debiera tener una regulación más detallada en procedimiento y características.

- La remisión por la universidad afectada de una memoria comprensiva de sus actividades docentes e investigadoras es excesivamente generalista, resulta confusa sobre el modo en que las administraciones van a ejercer las facultades inspectoras y da lugar a que la aplicación del procedimiento sea diferente según las CCAA y, en consecuencia, discrimina a las universidades. Debiera señalarse cómo deben las CCAA ejercer las competencias. Cuáles serían los mecanismos que evidencien los posibles incumplimientos y el plazo de ejecución. La falta de regularización del funcionamiento de las competencias y de control, por otra parte, lleva a alejar al sistema universitario de la pretendida calidad y de la credibilidad que se pretenden conseguir. 
"Centros que impartan enseñanzas conducentes a la obtención de títulos extranjeros”. (Art. 12)

- Este artículo necesitaría una mayor aclaración sobre todo en lo que concierne a los requisitos establecidos.

Efectos de autorización (Art. 13)

- Debiera incluir también a los centros adscritos y sus requisitos, a fin de evitar el vacío legal que quedaría al derogar el RD 557/91.

Acreditación institucional de centros (Art. 14)

1. La ANECA o los órganos de evaluación de las CCAA, inscritos en el EQAR, a solicitud de la universidad, emiten el informe de evaluación favorable. El Consejo de Universidades dicta y envía la resolución de acreditación a la universidad, a la Comunidad Autónoma y al Ministerio, a los efectos de inscripción de los centros acreditados en el Registro de Universidades, Centros y Titulaciones.

\section{Requisitos:}

1. Haber renovado la acreditación oficial de al menos la mitad de los títulos oficiales de grado y máster que impartan.

2. Contar con la certificación de la implantación de su sistema de garantía interno de calidad.

3. Si un título, o más, se imparte en varios centros de la misma universidad, no se puede solicitar la acreditación de dichos centros hasta que se renueve la acreditación del título(s).

4. El certificado de implantación de su sistema de garantía interno de calidad podrá ser expedido por la ANECA u otra agencia de las CCAA inscrita en la EQAR.

5. La renovación de la acreditación de los centros se deberá producir antes del transcurso de los 5 años contados a partir de la fecha de obtención de la primera resolución de acreditación. La solicitud deberá incorporar un informe de un panel de expertos externos e independientes. La evaluación la realizará la ANECA u otra de las CCAA inscrita en la EQAR.

6. Si la resolución del Consejo de Universidades es desestimatoria, el plazo para renovación de la solicitud no será superior a un año desde la fecha de la resolución. 
- Este artículo regula la acreditación institucional de los centros de una universidad. En general, se puede aceptar con un juicio positivo, ya que puede servir para reducir la burocracia asfixiante de estos procesos sin merma de la garantía de la calidad universitaria.

- No obstante, al exigirse que al menos se haya acreditado individualmente la mitad de los títulos del centro cuya acreditación institucional se pretende, durante un período de tiempo no desdeñable va a implicar una multiplicación de procesos (los de los títulos en sí y además los de los centros). Si al final se quieren reducir los procesos (o al menos el aumento desmedido de recursos destinados a éstos), debería fomentarse esta acreditación sin esperar a acreditar la mitad de los títulos del centro (por ejemplo, si la Universidad en su conjunto ya ha acreditado un número significativo de los títulos que imparte).

- Por otro lado, parece incorrecto que la no acreditación del centro implique la necesidad de que la Universidad solicite la renovación de la acreditación de todos sus títulos; este texto debe matizarse indicando que afecta exclusivamente al Centro y a los títulos cuya oficialidad desee mantenerse, y todo ello sin perjuicio de las re-acreditaciones de la mitad de los títulos del Centro cuya acreditación ya fue concedida antes de solicitar la acreditación institucional.

- Y, como se ha indicado, para evitar la confusión de que las agencias de evaluación participan de las funciones de supervisión y control de las Universidades que incumben exclusivamente a las Administraciones educativas, debería regularse la llamada acreditación institucional íntegramente en el RD regulador de la ordenación de las enseñanzas universitarias, pues es en esa instancia donde se regulan las consecuencias de esta acreditación.

\section{Disposiciones adicionales}

Adicional primera. Adaptación de las Universidades y centros universitarios a los requisitos previstos en este Real Decreto

Plazo para cumplir los requisitos, personales, de infraestructura y medios materiales, 3 años.

Las universidades ya creadas deberán presentar el plan al que se refiere el art. 5.3.: cuando quieran implantar titulaciones de un rama en la que no tienen oferta.

Los centros que imparten enseñanzas universitarias con arreglo a sistemas educativos extranjeros disponen de 3 años para su adaptación. 
- Convendría ampliar a cuatro o cinco años la duración del período de adaptación al nuevo RD, que es la duración normal de un estudio universitario de grado en la actualidad.

- Llama la atención que no se regulen los centros adscritos, ni en su creación ni en su adscripción misma, a semejanza del RD precedente.

\section{Comentarios finales}

Como ya se ha visto en el articulado, son diversas las razones por las que se propone que este Borrador debería ser revisado y matizado tanto en su conjunto como en su articulado, para que dé respuesta apropiada a su denominación y objeto, ya que, además de la creación, pretende la supervisión y control de las universidades y no la mera acreditación de títulos. En definitiva, la introducción de estos aspectos resulta confusa y requeriría una regulación integral del RD 1393/2007 en orden a la regulación de las enseñanzas oficiales.

El objetivo que pretende alcanzar en torno a la Ley de unidad de mercado, que se expone en el preámbulo y que está avalado por el Informe de la Comisión de Expertos, designada por el Ministerio de Educación para la Reforma del Sistema Universitario, a tenor de lo que queda reflejado en el articulado, no parece que pueda ser alcanzado. Este Borrador precisa de una mayor coherencia con la norma fijada y de medidas imaginativas para que el sistema universitario resulte cohesionado y orientado, desde la cualidad y la especialización, hacia la calidad y su mejora. Convendría, por lo demás, un mayor énfasis y desarrollo de la normativa.

En este contexto, consideramos que la modificación de un Real Decreto con casi 25 años de trayectoria, dedicado primariamente a la regulación de la creación o reconocimiento de Universidades y Centros de Enseñanza Superior y de su supervisión y control, debería pretender algo más que la adaptación a los textos legales y reglamentarios que, en esta materia, se han prodigado con posterioridad. El proyecto de Real Decreto reproduce en gran medida el articulado de su antecedente, tal vez intensificando, con mayor o menor grado de justificación, sus requisitos y condiciones, determinando un alto grado de uniformidad en las características requeridas a las Universidades. Cabría preguntarse, sin embargo, si las transformaciones que han ido aconteciendo en el ámbito universitario (y que ya reseña el Preámbulo de la Ley Orgánica de Universidades de 2001) no demandarían una respuesta más imaginativa que permitiera un sistema universitario español ciertamente vertebrado, cohesionado y orientado a la calidad y mejora continua, pero también desde la cualidad y la especialización. Para ello habría que tener en cuenta también la llamada de atención 
(señalada en el Informe de la Comisión de Expertos designada por el Ministerio de Educación para la Reforma del Sistema Universitario Español) en pro de la necesidad de reformar -también en su regulación jurídica- el sistema universitario español para, entre otros fines, servir de impulso al desarrollo social y económico de España mediante la excelencia, la diferenciación y la especialización. Nos podríamos preguntar también por la vigencia de la Ley 20/2013, de 9 de diciembre, de garantía de la unidad de mercado (citada expresamente en el Preámbulo del proyecto de Real Decreto).

Por otro lado, debería aclararse con mayor nitidez el objeto de este RD. Tal como se ha visto, en los art. 1 y 11 no se indica cómo se ejercen las competencias de las Administraciones educativas, ni los mecanismos con arreglo a los cuales se apreciarían los posibles incumplimientos de la Universidad, ni en qué momento se efectuarían las revisiones. Esta exigencia se contrae a reclamar la remisión por parte de la Universidad de una memoria comprensiva de sus actividades docentes e investigadoras. El ejercicio cabal de estas funciones es, en efecto, indispensable para sostener la solidez y credibilidad del sistema universitario y para evitar el alejamiento de la búsqueda de la calidad. Por este motivo, resulta preocupante que no se detalle el funcionamiento de las competencias de supervisión y control de las Universidades por parte de las Administraciones educativas.

Se crea una nueva figura de acreditación institucional de Centros (capítulo IV), que presenta rasgos positivos, pero sorprende que se regule en este RD, puesto que podría estimarse que constituye una forma más o menos solapada de llevar a cabo las funciones de supervisión y control, aunque la acreditación institucional de Centros se basa -si nos ceñimos a la letra del RD- en criterios diferentes a los exigidos para la creación y continuidad de una Universidad. En suma, podría parecer que las facultades inspectoras y la comprobación de los requisitos demandados se derivan en el RD hacia otra instancia, como son las Agencias de Evaluación, cuyo informe es determinante para la acreditación de un centro y que, sin embargo, carecen manifiestamente de competencia para el ejercicio de funciones de supervisión y control.

Finalmente, a modo de síntesis, la notoria presencia de las Agencias de Evaluación queda remarcada en:

- La evaluación del plan académico de la creación de una Universidad; la implantación de titulaciones en una rama donde no existe oferta en la Universidad.

-La evaluación de los títulos extranjeros; y, como se ha indicado, en la acreditación institucional de Centros. 
Ni por razones técnico-jurídicas (su naturaleza jurídica), ni por razones prácticas (los medios disponibles), parece conveniente que la intervención de estas agencias de evaluación deba ser tan decisiva cuando se trata de la creación o el reconocimiento de Universidades y de la supervisión de su actividad.

Por el contrario, se echa en falta que estas funciones de supervisión y control encomendadas a las Administraciones educativas (y que no pueden desempeñar las agencias de evaluación) sean reguladas de manera más precisa y garantista en cuanto a su procedimiento, habida cuenta de las consecuencias radicales que de su ejercicio se pueden derivar. De esta forma, un ejercicio real y ponderado de estas funciones permitiría aumentar la confianza en el buen funcionamiento del sistema universitario.

Finalmente, tal como se indicaba en el art. 1, se solicita la regulación íntegra de todos los aspectos que aborda este RD para que alcance los objetivos propuestos ${ }^{1}$.

1 Para la elaboración de este informe se han tenido en cuenta los comentarios al Borrador hechos por las

Universidades de Comillas y Deusto (2014). 